\title{
Surface fields on the source-excited dielectric wedge
}

\section{Balling, $\mathbf{P}$}

\section{Published in:}

I E E E Transactions on Antennas and Propagation

Publication date:

1973

\section{Document Version}

Publisher's PDF, also known as Version of record

Link back to DTU Orbit

\section{Citation (APA):}

Balling, P. (1973). Surface fields on the source-excited dielectric wedge. I E E E Transactions on Antennas and Propagation, 21(1), 113-115.

\section{General rights}

Copyright and moral rights for the publications made accessible in the public portal are retained by the authors and/or other copyright owners and it is a condition of accessing publications that users recognise and abide by the legal requirements associated with these rights.

- Users may download and print one copy of any publication from the public portal for the purpose of private study or research.

- You may not further distribute the material or use it for any profit-making activity or commercial gain

- You may freely distribute the URL identifying the publication in the public portal

If you believe that this document breaches copyright please contact us providing details, and we will remove access to the work immediately and investigate your claim. 
[3] T. Soejima, "Passive repeater of the second kind using double flat reflectors," J. Inst. Elec. Commun. Eng. Jap., vol. 42, pp. 22-27, Tay 1959 .

[4] S. Takeshita, "Lommel's function with two variables, real and complex," J. Inst. Elec. Commun. Eng. Jap., vol. 46, pp. 89-90, Oct. 1963 .

[5] A. Sommerfeld, Optics. New York: Academic Press, 1964, pp. $216-221$.

\section{Surface Fields on the Source-Excited Dielectric Wedge}

\section{PETER BALLING}

Abstract-Approximate surface fields due to a plane-wave solution and a local-mode solution are compared. The plane-wave solution, which is new, is shown to agree well with experiment. The local-mode solution, which often has been applied to tapered waveguides and antennas, fails near the cutoffs of the surface waves.

\section{INTRODCCTION}

For an open waveguide which acts as a surface-wave antenna, it is well known that a tapering can improve radiation pattern and antenna impedance considerably over a wide frequency band, e.g., the tapered dielectric rod antenna and the log-periodic antenna. In such cases, the field along the tapered structure is often approximated with either a surface or a leaky wave which varies in accordance with the transverse cross section of the tapered waveguide.

In this communication such a local-mode solution for the sourceexcited dielectric wedge is compared with a more accurate solution in terms of plane waves. A segment of the wedge, the tapered dielectric slab waveguide, has been considered by Marcuse [1] and Shevchenko [2] as a transition between two slab waveguides of different width. They obtained an approximate solution in terms of a local surface wave and also considered the radiation losses. A similar work has been carried out by Bahar [3].

\section{Plane-Wave Solution}

The dielectric wedge is excited by a magnetic, unit line current at the plane of symmetry (Fig. 1). As shown in [4], an accurate, although still approximate solution, can be constructed by expanding the field from the line current in an angular spectrum of plane waves which then are multiply reflected at the plane interfaces of the wedge. This procedure leads to the following approximation to the magnetic field, $H=H_{y} \hat{y}$, at the interfaces

$$
\begin{aligned}
H_{y}(z, d)=-\frac{\omega \epsilon \epsilon_{0}}{4 \pi} \sum_{n=0}^{N} \int_{-z / 2+i \infty}^{-/ 2-i \infty} T(w) \prod_{n=1}^{n} R(w+2 \nu \psi) \\
\cdot \exp \left(i k_{1} \rho_{n} \cos \left(w-\theta_{n}\right)\right) d^{3} w .
\end{aligned}
$$

The time factor $\exp (-i \omega t)$ has been suppressed. The TM Fresnel reflection and transmission coefficients $R(w)$ and $T(w)=1+R(w)$ are defined through

$$
R(w)=\frac{\cos w-\left(\epsilon-\epsilon^{2} \sin ^{2} w\right)^{1 / 2}}{\cos w+\left(\epsilon-\epsilon^{2} \sin ^{2} w\right)^{1 / 2}}
$$

The expansion variable $w$ is formally the final angle of incidence of the plane waves and $k_{1}$ is the wavenumber of a plane wave in the dielectric. The first term in the summation gives the direct illumination, the second term the once reflected illumination, the third term the twice reflected illumination, and so on. Only $N+1$ terms contribute to the surface field because all waves in the wedge, at

Manuscript received May 22, 1972 ; revised July 24, 1972. This work was supported by the Danish Government Fund for Scientific and Industrial Research.

The author was with the Laboratory of Electromagnetic Theory, Technical Lniversity of Denmark, Lyngby, Denmark. He is now with the Department of Electrical Engineering. University of Zambia,
Lusaka, Zambia.

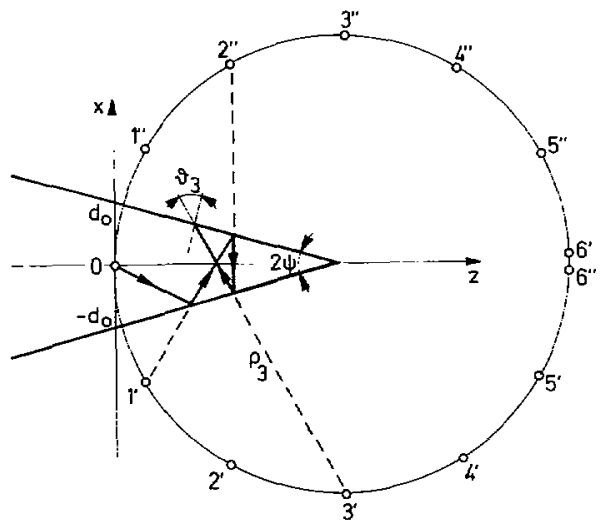

Fig. 1. Dielectric wedge excited by line current at 0 . Ray path is constructed by means of the images which are situated on circle with center at tip.

least after

$$
N=\left[\frac{\pi-\psi}{2 \psi}\right]
$$

reflections, disappear towards the broad end of the wedge. This can be seen for $N=5$ on Fig. 1. This figure also shows for $n=3$ that $\rho_{n}$ and $\theta_{n}$ are the path length and the final angle of incidence of the $n$ times reflected ray. For small wedge angle and moderate dielectric constant, the number of partial reflections can be large. Then the summation in (1) converges much faster than (3) predicts. The integrals in (1) are evaluated by numerical integration along paths of steepest descents in angular $w$ plane. Thus each term contributes with one saddle-point wave which becomes equal to a geometric-optical ray in the high-frequency limit. If this ray hits the interfaces at angles which are greater than the critical angle, a corresponding number of lateral waves contribute in addition to the saddle-point wave. Lateral waves are due to diffraction of critically incident waves [5]. In (1), a lateral wave appears when the path of steepest descents crosses a branch point due to a square root in one of the Fresnel coefficients.

As no exact numerical results are available for the dielectric wedge, we have to resort to experiments in order to obtain a verification of the plane-wave approach. In the experiment that will be described, the $K$-band parallel-plate instrument at the Polytechnic Institute of Brooklyn was employed to simulate the two-dimensional wedge. The excitation was a current probe which produces a TE field. Hence, the dual form of (1) must be employed. Also, as the electric field was measured along a path a small distance $a$ from the wedge surface, the plane-wave spectra include a factor,

$$
D(w)=\exp \left(i k a\left(1-\epsilon \sin ^{2} w\right)^{1 / 2}\right) .
$$

This exponential transfers each plane-wave component of the surface field to the distance $a$ from the wedge surface.

The experiment was carried out at $25 \mathrm{GHz}$. On Fig. 2, the electric field measured approximately $2 \mathrm{~mm}$ away from the interface is compared with electric fields calculated 1 and $2 \mathrm{~mm}$ away. The curves indicate that $a \simeq 2 \mathrm{~mm}$ at the source and that $a \simeq 1 \mathrm{~mm}$ at the tip. The remaining small discrepancies can be attributed to various reflections which were present in the experimental setup. Thus the plane-wave result is within the experimental error equal to the measured surface field. As shown in [4], similar good agreement exists between the measured and the calculated radiation field. The latter is obtained by a numerical surface integration of the plane-wave wedge field.

\section{LOCAL-MODE SOLUTION}

The lacal-mode solution is derived from the well known exact solution to the $2 d_{0}$-wide dielectric slab excited by a magnetic, unit line current at the plane of symmetry. The magnetic field on 


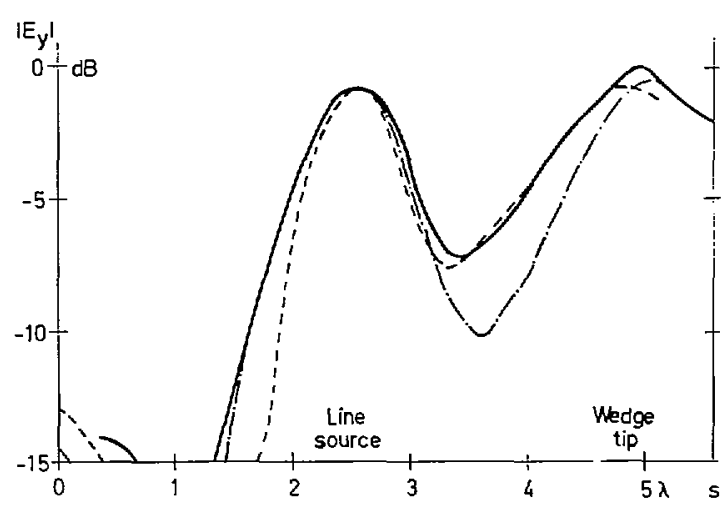

Fig. 2. Calculated and measured near fields versus distance along Whedge surface. Dielectric constant is $\epsilon=2.53$. Wedge angle is $2 \psi=11.9^{\circ}$. Normalized halfwidth at source
ured approximately $2 \mathrm{~mm}$ away. $=1.66 . \overline{\mathrm{mm}}$ Mwasured approximately $2 \mathrm{~mm}$ awa

the slab interface is determined by

$$
H_{y}\left(z, d_{0}\right)=-\frac{\omega \epsilon \epsilon_{0}}{4 \pi} \int_{-\infty}^{\infty} \frac{\exp (i \zeta z)}{\epsilon \kappa \cos \left(\kappa_{1} d_{0}\right)-i_{\kappa_{1}} \sin \left(\kappa_{1} d_{0}\right)} d \zeta .
$$

The variable of integration is the longitudinal wavenumber $\zeta$. The transverse wavenumbers fulfil

$$
\begin{aligned}
\kappa & =\left(k^{2}-\zeta^{2}\right)^{1 / 2} \\
\kappa_{1} & =\left(k_{1}{ }^{2}-\zeta^{2}\right)^{1 / 2}
\end{aligned}
$$

where $k$ and $k_{1}$ are the wavenumbers in free space and in the dielectric, respectively. The usual branch cuts are introduced for $\kappa$, i.e., Im $\kappa \geq 0$ in the upper Riemann sheet. It is well known that (5) can be changed to an expansion in transverse modes. This expansion consists of a continuous spectrum, the space wave, due to an integration around the branch cut of $\kappa$ and a discrete spectrum of surface waves.

The space wave contains the reactive fields at the source and the radiation fields. Along the interface it is significant only close to the source. We assume that this slab space wave approximates the corresponding fields on the wedge.

The surface waves are modified so that their phase velocities vary with the width of the wedge. We employ the principle of phase accumulation and neglect the losses of a surface wave due to reflection or due to scattering into either other surface waves or the continuous spectrum. With these assumptions, the power of a surface wave is constant along the wedge. When a surface wave directed towards the tip reaches cutoff, its amplitude becomes equal to zero as the finite power then is spread uniformly across the infinite cross section. The preceding approximations lead to the expression which was obtained by Shevchenko as a first approximation to a surface wave along a tapered dielectric slab waveguide [2].

\section{COMPARISONS}

Fig. 3(a) shows the amplitude of the surface field between the source and the wedge tip. As $k d_{0}=3.21$, two surface waves exist near the source. The slab space wave, the two modified surface waves as well as the sum, the local-mode field, and the plane-wave field have been plotted.

The cutoff of the fundamental surface wave SW1 creates a deep minimum in the local-mode field at the tip. Thus the local-mode solution can not produce the field maximum which was measured at the tip on Fig. 2. Close to cutoff, the longitudinal wavenumber of the surface wave exceeds only slightly the free-space wavenumber. Hence, there is a strong scattering from the surface wave into the fast radiation waves which essentially radiate towards endfire, i.e., the cutoff creates a "hot region" with strong radiation. The planewave solution takes this scattering into account. If forward seattering

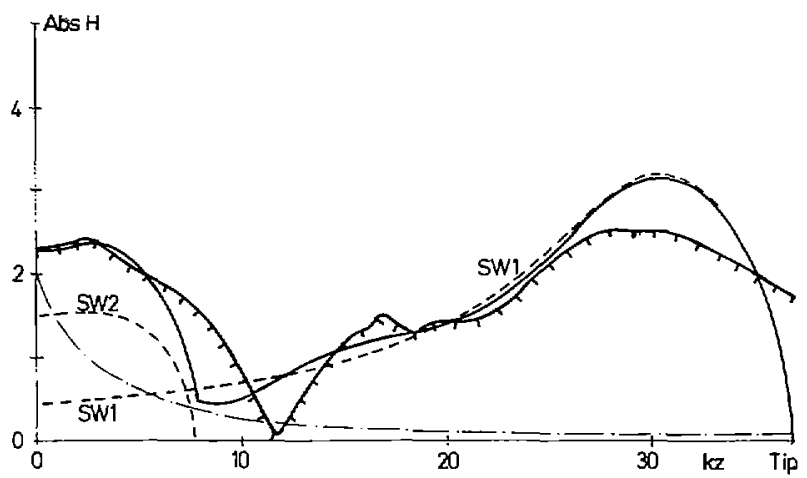

(a)

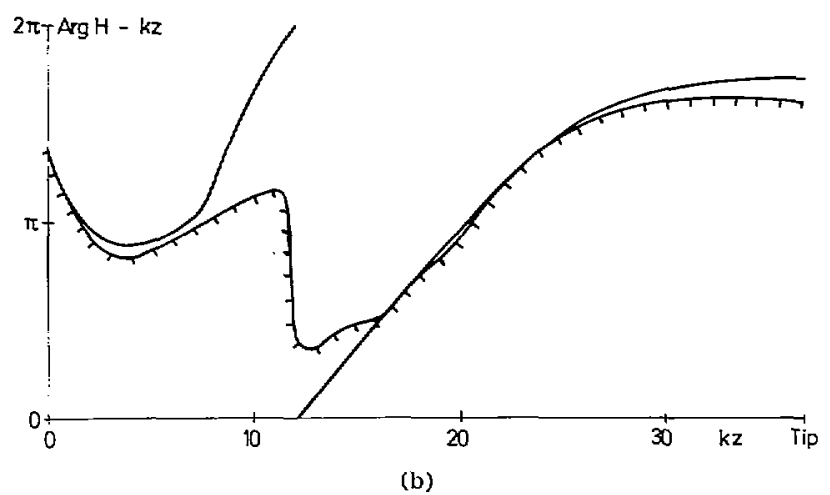

Fig. 3. (a) Amplitude of magnetic field along wedge surface. (b) Phase of magnetic field along wedge surface. Factor $-\omega_{\epsilon_{0}} / 4 \pi$ has been omitted and phase of plane wave along wedge has been subtracted Abscissa gives normalized distance along interface from line source towards tip. Dielectric constant is $\epsilon=2.53$. Wedge angle is $2 \psi=10^{\circ}$. Normalized balfwidth at source $k d_{0}=3.21$. - - Surface waves: SW1 and SW2. - - Slab space wave. - - Total local-mode field. TTT Plane-wave field.

dominates, the surface field will only be neglibly affected by diffraction at the tip and the plane-wave approach leads to accurate results. When backward scattering is significant, e.g., for large wedge angle and/or large dielectric constant, the scattered field still appears in the plane-wave solution. The result may then, however, be in error because of the omission of diffraction.

A second evidence of the strong forward scattering at cutoff is available on Fig. 3(a). Beyond the cutoff of the higher order mode SW2, we note a few dampened oscillations in the plane-wave field. These oscillations are due to interference between the slow fundamental surface wave $\mathrm{SW} 1$ and the fast radiation waves. The rate of oscillation indicates that the main part of the scattered field has a longitudinal wavenumber very close to $k$.

The phases of the local-mode field and the plane-wave field are compared on Fig. 3(a). The phase of a plane wave along the wedge has been subtracted. It is seen that the phase accumulation inherent in the local-mode approximation is quite accurate except near the cutoffs.

Although the differences on Fig. 3 between the plane-wave field and the loeal-mode field appear relatively insignificant, the fast surface-field components play an important role in the radiation process. Thus the plane-wave solution leads to much better agreement with measurements than the local-mode solution when the surface field is integrated numerically along the wedge interfaces to obtain the radiation field.

\section{Conclusion}

Away from the cutoffs of the surface waves, the local-mode solution agrees well with the more accurate, but at low frequencies also more complicated plane-wave solution. The local-mode solution should be avoided when cutoff regions with strong radiation occur. 
As the frequency increases, the local-mode solution becomes more complicated because more surface waves then contribute. The plane-wave solution, however, becomes steadily more efficient and tends towards the geometric-optical solution.

\section{ACKNOWLEDGMENT}

The author wishes to express his gratitude to Dr. Techn. J. Bach Andersen of the Laboratory of Electromagnetic Theory, the Technical University of Denmark, who supervised the work. The surfacefield measurement was carried out at the Polytechnic Institute of Brooklyn in cooperation with Dr. S. J. Maurer. The author is grateful for this valuable support.

\section{References}

[1] D. Marcuse, "Radiation losses of tapered dielectric slab waveguides," Bell Syst. Tech. J,, vol. 49, pp. 273-290, 1970.

[2] V. Y. Shevchenko, Continuous Transitions in Open Waveguides. Boulder, Colo.: Golem Press, pp. 125-138, 1971.

[3] E. Bahar, "Propagation of radio waves over a nonuniform layered medium," Radio Sci., vol. 5, pp. 1069-1079, 1970.

[4] P. Balling, "Radiation from the dielectric wedge," Licentiate thesis LD 20, Lab. Electromagn. Theory, Tech. Univ, Denmark, DK-2800 Lyngby, Denmark, 1971.

[5] L. MI. Brekhovskikh, Waves in Layered Media. New York: Academic Press, pp. 270-292, 1960 .

\section{An Alternate Frill Field Formulation}

\section{CHALMERS M. BUTLER AND LEONARD L. TSAI}

$A b s t r a c t-A n$ alternate form [1] for the near zone $E_{z}$ fields of an annular ring of magnetic current is derived. The new expression avoids numerical differentiation, offers significant improvements in efficiency, and is particularly useful in the analysis of coazially driven parallel arrays.

In [1] the near fields for an annular ring of magnetic current (frill) are obtained by applying numerical differentiation to the electric vector potential $F$ which, for the rotationally symmetric frill, possesses only a nonzero $\phi$ component $F_{\phi}$. If one proceeds with the vector potential formulation in a direct manner, certain simplifications result in the $E_{z}$ component of the field so that numerical differentiation can be avoided and one is able to obtain $E_{\varepsilon}$ everywhere by evaluating numerically a rather simple integral. Knowledge of $E_{z}$ is of particular importance in analyses of coaxialaperture driven arrays of parallel monopoles above a ground plane.

For the magnetic frill current [see Fig. 1]

$$
M^{\prime}=\hat{u}_{\phi^{\prime}} \frac{-1}{\rho^{\prime} \ln (b / a)}
$$

the electric vector potential is given by

$$
F(\boldsymbol{r})=\frac{\epsilon}{4 \pi} \iint_{\text {frill surfaoe }} M^{\prime}\left(\boldsymbol{r}^{\prime}\right) \frac{\exp \left(-j k\left|r-r^{\prime}\right|\right)}{\left|r-r^{\prime}\right|} d s^{\prime}
$$

with the corresponding electric field given by

$$
E=-\frac{1}{\epsilon} \operatorname{curl} F
$$

Manuscript receiyed May 30, 1972; revised September 5, 1972. This work was supported in part by NSF Grant GU/3833.

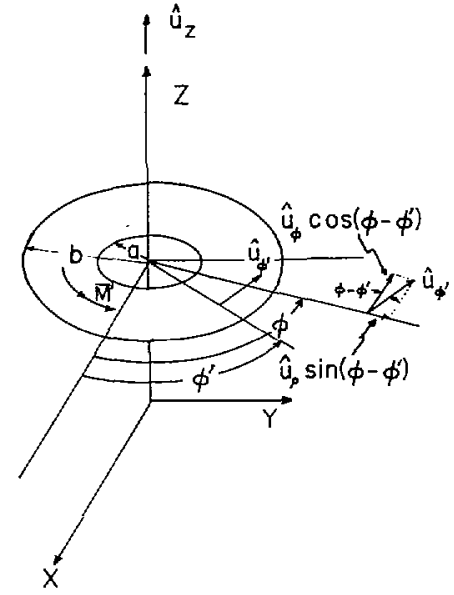

Fig. 1. Frill geometry.

When the magnetic frill is parallel to the $x y$ plane and concentric with the $z$ axis and the geometry of the problem is as depicted in Fig. 1, (1) reduces to

$F(\rho, \phi, z)=-\frac{\epsilon}{4 \pi \ln (b / a)} \int_{\rho^{\prime}=\alpha}^{b} \int_{\phi^{\prime}=0}^{2 \pi} \frac{\hat{u}_{\phi^{\prime}}\left(\phi^{\prime}\right)}{\rho^{\prime}} \frac{\exp (-j k R)}{R} \rho^{\prime} d \phi^{\prime} d \rho^{\prime}$

where

$$
R=\left[\rho^{2}+\rho^{\prime 2}-2 \rho \rho^{\prime} \cos \left(\phi-\phi^{\prime}\right)+\left(z-z^{\prime}\right)^{2}\right]^{1 / 2}
$$

It is to be noted that the source is in the $\phi^{\prime}$ direction and has a projection onto the observation coordinates implied by

$$
\hat{u}_{\phi^{\prime}}=\hat{u}_{\rho} \sin \left(\phi-\phi^{\prime}\right)+\hat{u}_{\phi} \cos \left(\phi-\phi^{\prime}\right)
$$

where the terms in (5) are defined in Fig. 1. Thus from (3)

$$
F_{\phi}=-\frac{\epsilon}{4 \pi \ln (b / a)} \int_{\rho^{\prime}=a}^{b} \int_{\phi^{\prime}=0}^{2 \pi} \cos \left(\phi-\phi^{\prime}\right) \frac{\exp (-j k R)}{R} d \phi^{\prime} d \rho^{\prime}
$$

and from (2) the $z$ component of the electric field is seen to be

$$
E_{z}=\left(-\frac{1}{\epsilon} \operatorname{curl} F\right) \cdot \hat{\iota}_{z}=-\frac{1}{\epsilon}\left[\frac{\partial F_{\phi}}{\partial \rho}+\frac{1}{\rho} F_{\phi}\right]
$$

since $F_{\rho}=0$ everywhere. Using the form of $F_{\phi}$ given in (6), one obtains the derivative needed in (7):

$$
\begin{aligned}
\frac{\partial F_{\phi}}{\partial \rho}=-\frac{\epsilon}{4 \pi \ln (b / a)} \int_{\rho^{\prime}=a}^{b} \int_{\phi^{\prime}=0}^{2 \pi} \cos \left(\phi-\phi^{\prime}\right) \\
\cdot \frac{\partial}{\partial R}\left[\frac{\exp (-j k R)]}{R}\right] \frac{\partial R}{\partial \rho} d \phi^{\prime} d \rho^{\prime} .
\end{aligned}
$$

One integration of (6) by parts with respect to $\phi^{\prime}$ and the observation that both $\sin \left(\phi-\phi^{\prime}\right)$ and $R$ are periodic in $\phi^{\prime}$ with period $2 \pi$ enable one to write $F_{\phi}$ in the following form:

$F_{\phi}=-\frac{\epsilon}{4 \pi \ln (b / a)} \int_{\rho^{\prime}=a}^{b} \int_{\phi^{\prime}=0}^{2 \pi} \sin \left(\phi-\phi^{\prime}\right) \frac{\partial}{\partial R}\left[\frac{\exp (-j k R)}{R}\right] \frac{\partial R}{\partial \phi^{\prime}}$ $\cdot d \phi^{\prime} d \rho^{\prime}$ 